\title{
Basin Hopping with Occasional Jumping
}

\author{
Masao Iwamatsu ${ }^{1}$ \\ Department of Physics, General Education Center, Musashi Institute of \\ Technology, Setagaya-ku, Tokyo 158-8557, Japan \\ Yutaka Okabe \\ Department of Physics, Tokyo Metropolitan University, Hachioji, Tokyo 192-0397, \\ Japan
}

\begin{abstract}
Basin-Hopping (BH) or Monte-Carlo Minimization (MCM) is so far the most reliable algorithms in chemical physics to search for the lowest-energy structure of atomic clusters and macromolecular systems. BH transforms the complex energy landscape into a collection of basins, and explores them by hopping, which is achieved by random Monte Carlo moves and acceptance/rejection using the Metropolis criterion. In this report, we introduce the jumping process in addition to the hopping process in BH. Jumping are invoked when the hopping stagnates by reaching the local optima, and are achieved using the Monte Carlo move at the temperature $T=\infty$ without rejection. Our Basin-Hopping with Occasional Jumping (BHOJ) algorithm is applied to the Lennard-Jones clusters of several notoriously difficult sizes. It was found that the probability of locating the true global optima using BHOJ is significantly higher than the original $\mathrm{BH}$.
\end{abstract}

Key words: Basin-Hopping, lowest-energy structure, Lennard-Jones cluster PACS: 02.60.Pn, 02.70.Tt, 36.40.Mr

\section{Introduction}

The Monte Carlo method based on the Metropolis algorithm is the most successful and influential stochastic algorithm of the 20th century and has been

1 E-mail: iwamatsu@ph.ns.musashi-tech.ac.jp, Tel: +81-3-3703-3111 ext.2382, Fax: $+81-3-5707-2222$

Preprint submitted to Elsevier Science

25 September 2018 
used in variety of applications not limited to physics [1]. The method is powerful in an exhaustive search of highly multi-dimensional phase space, and, hence, has been routinely used to calculate the thermal averaging of statistical physics.

Aside from the applications to statistical physics, the Metropolis algorithm has been used as a vehicle for global optimization, that is, a task to search for the lowest minimum point in a rugged landscapes in a high dimension. In fact, the simulated annealing (SA) [2] based on the Metropolis algorithm is the oldest metaheuristics in global optimization.

In global optimization, a good balance between a global search (exploration) and a local search (exploitation) is necessary. Since the Metropolis algorithm has only the ability to perform a global search, it is usually necessary to augment SA using a more traditional local optimization method to handle realistic problems [3]. Later, a method which combines the Metropolis algorithm and the gradient-based local search algorithm was proposed. That is the Monte Carlo minimization (MCM) of Li and Sherga [4], and the basin-hopping (BH) of Wales and Doye [5]. Wales and Doye [5], for example, used their BH algorithm to study the lowest-energy structures of Lennard-Jones clusters that consist of up to 110 atoms successfully.

In this paper, we propose a new variant of the Basin-Hopping(BH) algorithm. In contrast to another variant of the $\mathrm{BH}$ by Leary and Doye $[6,7]$ where only the down-hill search is allowed, we borrow the concept of extremal optimization [8] or thermal cycling [9], and introduce the process of jumping to enhance the search of a rugged energy landscape.

\section{Jumping in Basin Hopping}

The Basin Hopping (BH) algorithm (which is also called the Monte Carlo plus minimization, MCM) uses hopping due to Monte Carlo random walks to explore the phase space, and gradient-based local optimization to locate the local minimum or basin plus Metropolis criterion to accept or reject the move.

Since the energy landscape explored by the usual Monte Carlo move and immediate relaxation to a nearby basin of attractor using the gradient method in $\mathrm{BH}$ looks like hopping among basin of attractors, the algorithm is termed "Basin Hopping". In order to enhance the exploration, the Monte Carlo move in $\mathrm{BH}$ consists of a simultaneous displacement of all particles in the cluster in contrast to the usual Monte Carlo method in classical statistical mechanics [10] where usually a randomly chosen single particle is displaced. 


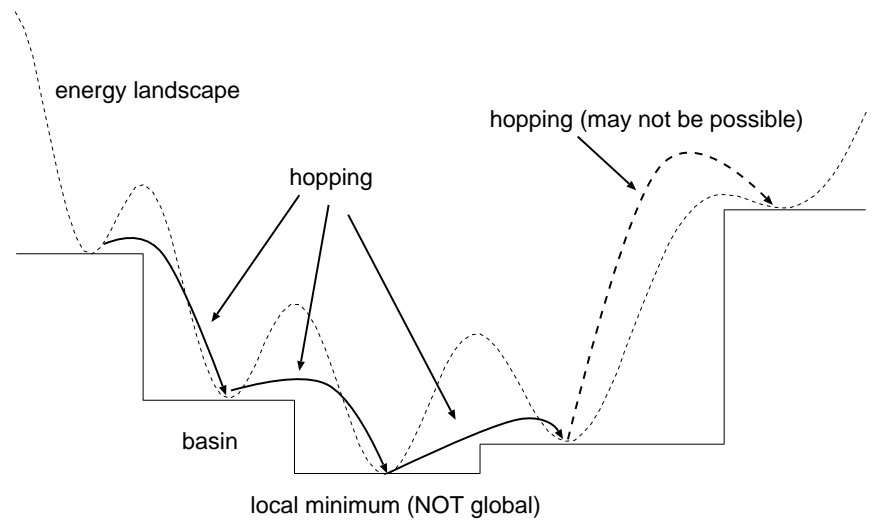

Fig. 1. The exploration of the energy landscape using the BH algorithm. The uphill moves, which uses the usual Monte Carlo move may not be possible.

The BH has been extensively tested [5] for the simplest benchmark problem for the Lennard Jones clusters $\mathrm{LJ}_{n}[3,11,12,13,14]$ where the total energy of $n$-atom cluster is given by

$$
E_{n}=2 \sum_{i=1}^{n} \sum_{j=1}^{n}\left(\frac{1}{r_{i j}^{12}}-\frac{1}{r_{i j}^{6}}\right)
$$

where $r_{i j}$ is the distance between two particles $i$ and $j$ within the cluster. Even for this simple Lennard-Jones problem, the BH clarified the extremely complex energy landscape for several clusters [15], and it even failed to locate the lowest-energy structures for several special sizes.

For example, the $\mathrm{BH}$ could not locate the lowest minimum of $\mathrm{LJ}_{75-77}, \mathrm{LJ}_{98}$, and $\mathrm{LJ}_{102-104}$ easily [5] when an unbiased search that starts from a completely random initial configuration of clusters is used. In fact, in order to find out the lowest-energy structure of $\mathrm{LJ}_{76}$, Wales and Doye [5] had to use seeding to feed the $\mathrm{BH}$ the lowest-energy structure of smaller $\mathrm{LJ}_{75}$ or larger $\mathrm{LJ}_{77}$ clusters. The unbiased search has an extremely low probability (4\%) of hitting the lowestenergy structure even when large numbers (100 times) of a fairly long run (5000 step) were executed. The same problem occurred for $\mathrm{LJ}_{102-104}$, and less severely in $\mathrm{LJ}_{69}, \mathrm{LJ}_{78}$ and $\mathrm{LJ}_{107}$.

Difficulty occurs when the energy landscape consists of several large valleys (funnels) instead of one, and the funnel corresponding to the lowest-energy minimum is narrow and separated from other funnels by a high barrier [15]. The Monte Carlo trial move plus local minimization and acceptance/rejection using the Metropolis criterion is less powerful and time-consuming to overcome such a large barrier (Fig. 1).

Recently, a new variant of the basin-hopping algorithm called the "Monotonic Sequence Basin-Hopping algorithm" (MSBH) was proposed by Leary and 


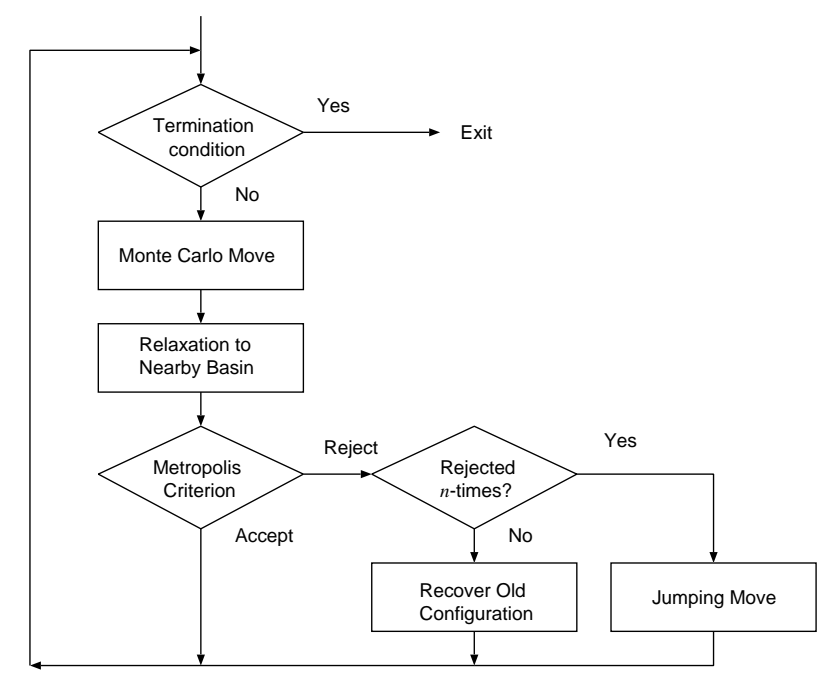

Fig. 2. The flow-chart of the basin-hopping with occasional jumping (BHOJ) algorithm.

Doye $[6,7]$. Their algorithm is essentially the $\mathrm{BH}$ at temperature $T=0$, which allows only downhill moves. When the search is stacked at a local minimum, the program restarts from a new random configuration. Therefore, MSBH $[6,7]$ is essentially the multi-start strategy of a greedy search. Naturally, the MSBH algorithm seems less powerful [7] than the original $\mathrm{BH}$ algorithm because it is not equipped with any mechanism to cross the barrier.

One of the present authors [16] suggested another extension of the BH by introducing the concept of "extremal optimization" (EO) proposed by Boettcher and Percus [8]. In this EO-based basin-hopping (EOBH) algorithm, the Monte Carlo move of only one less-bounded particle within the cluster is attempted, and every move is accepted without using the Metropolis criterion. Therefore this $\mathrm{EOBH}$ is essentially the $\mathrm{BH}$ at $T=\infty$. Although the $\mathrm{EOBH}$ can achieve the crossing of high barriers in contrast to the $\mathrm{MSBH}$, it is again less powerful than the original $\mathrm{BH}$ because its ability in terms of a local search is less effective though the method is proven to be useful to enumerate all the low-energy structures [16].

The lesson learned from these two previous exercises is that the inclusion of the high-temperature Monte Carlo move at $T=\infty$ will enhance the ability of a global search (exploration), but the original Metropolis criterion of the Monte Carlo move should be retained to maintain the efficiency of a local search (exploitation). Actually, such a prescription of re-heating or thermal treatment at high temperatures has been repeatedly proposed in the application of the simulated annealing (SA) [9,17]. For example, Möbius et al, introduced "Thermal Cycling" and Ingber [17] introduced "reannealing" in $\mathrm{SA}$. In order to enhance the ability of crossing the high barrier in the $\mathrm{BH}$, we have introduced a re-heating process called "jumping" as shown in Fig. 2. 


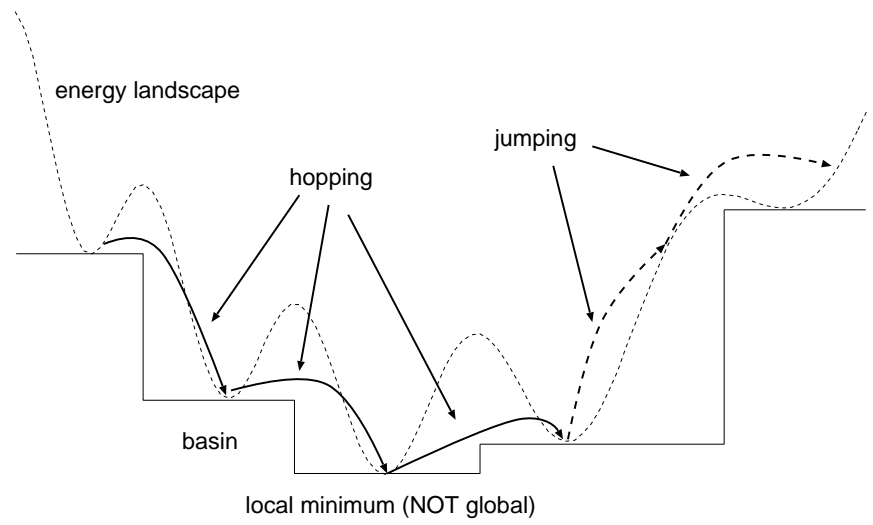

Fig. 3. The exploration of the energy landscape (dotted line) using the BHOJ algorithm. There are two kinds of uphill moves: one is the hopping (thick solid arrows) between basins (thin solid line) which uses the usual Monte Carlo move followed by the local minimization and Metropolis acceptance, another is the jumping (thick chain arrow) using a simple Monte Carlo move without minimization and rejection.

Jumping is the Monte Carlo move without relaxation (local minimization) at $T=\infty$, and, hence, it is always accepted. When the usual Monte Carlo moves are rejected MAX times, the system is judged to be trapped at the local minimum. Then the temperature is raised to $T=\infty$, and the Monte Carlo moves (jumpings) are executed JMP times in the search space to allow for the system to escape from the local minimum (Fig. 3). This move is always accepted irrespective of the increase in the energy because of $T=\infty$ and is called jumping here. The parameter MAX is used to detect the entrapment. The parameter JMP is used to try to climb up the barrier several times. If the JMP is too large, the algorithm is nothing more than simple random multi-start strategy. It is essential to keep the partial memory of the previous state [9]. So, the JMP should not be too large. We will call this version of $\mathrm{BH}$ the "Basin-Hopping with Occasional Jumping" (BHOJ).

Now, the exploration of phase space in our BHOJ proceeds as follows (Fig. $3)$ : the rugged energy landscape is transformed into successive steps of the basin by local minimization. The downhill move is simply the descending of the stairs by hopping. There are, however, two kinds of uphill moves. One is by climbing the stairs by hopping using the Metropolis criterion, which is costly and may not be effective to climb up the high barrier because the moves with a large energy difference are rejected by the Metropolis criterion. Another move is jumping, which does not use local minimization and is always accepted, so the uphill moves by jumping do not use stairs and are simply along the surface of the hill (Fig. 3). This jumping must be an efficient way to escape from a local minimum (valley) and to explore the next basin of the valley when it is separated by high barriers. 
Table 1

Success rates of original BH [5], MSBH [7], PFA [20] and our BHOJ for selected Lennard-Jones clusters which are notoriously difficult cases to find out the lowestenergy structure. In MSBH Leary conducted experiments 1000 times while we conducted experiments 100 times for original BH and BHOJ.

\begin{tabular}{crrrrr}
\hline Cluster & Energy & BH & MSBH & PFA & BHOJ (JMP) \\
\hline $\mathrm{LJ}_{38}$ & -173.928427 & $87 / 100$ & $124 / 1000$ & $39 / 100$ & $96 / 100(7)$ \\
$\mathrm{LJ}_{75}$ & -397.492331 & $1 / 100$ & $4 / 1000$ & $2 / 200$ & $5 / 100(3)$ \\
$\mathrm{LJ}_{76}$ & -402.894866 & $5 / 100$ & $8 / 1000$ & $2 / 50$ & $10 / 100(5)$ \\
$\mathrm{LJ}_{77}$ & -409.083517 & $6 / 100$ & & $1 / 50$ & $5 / 100(7)$ \\
$\mathrm{LJ}_{98}$ & -543.665361 & $10 / 100$ & $6 / 1000$ & $4 / 100$ & $10 / 100(3)$ \\
$\mathrm{LJ}_{102}$ & -569.363652 & $3 / 100$ & $31 / 1000$ & $9 / 100$ & $16 / 100(3)$ \\
$\mathrm{LJ}_{103}$ & -575.766131 & $3 / 100$ & & $3 / 30$ & $13 / 100(5)$ \\
$\mathrm{LJ}_{104}$ & -582.086642 & $3 / 100$ & & $2 / 30$ & $12 / 100(3)$ \\
\hline
\end{tabular}

\section{Experiments}

In order to test the performance of our modification of the $\mathrm{BH}$ with occasional jumping (BHOJ), we have calculated the lowest-energy structure of the Lennard-Jones clusters of a particular size, $\mathrm{LJ}_{38}, \mathrm{LJ}_{75-77}, \mathrm{LJ}_{98}$, and $\mathrm{LJ}_{102-104}$ for which the original $\mathrm{BH}$ is not effective. We conducted a 100 independent unbiased search starting from 100 random initial structures with the maximum of 5000 iterations which do not include the jumping process. We also performed the same experiment using the original $\mathrm{BH}[18]$ with the same random initial structures. The temperature is fixed at $T=0.8$. Two additional parameters MAX and JMP for the BHOJ are arbitrarily fixed to MAX $=10$ and $\mathrm{JMP}=3$ or 5 or 7 .

Table 1 gives the success rates of 100 unbiased searches. Our BHOJ could successfully reproduce the lowest-energy structures in the literature $[5,19]$. It is apparent that our BHOJ performs in general better than the original $\mathrm{BH}$. The success rate increased twice to five times from the original $\mathrm{BH}$ to the BHOJ. Thus, the ability of exploration is in fact enhanced by the introduction of jumping processes. For the sake of comparison, we also showed the results of MSBH of Leary [7] and the parallel fast annealing (PFA) of Cai et al. [20].

Figure 4 shows the trajectory of a successful run for $\mathrm{LJ}_{102}$ when using the BHOJ. Jumping occurs only 9 times during the run of 0 to 2500 steps. However, this jumping may induce the uphill moves which assist in the exploration of the next valley separated from the previous valley by a high barrier, and our BHOJ can successfully find out the lowest-energy structure at approximately 
1400 steps.

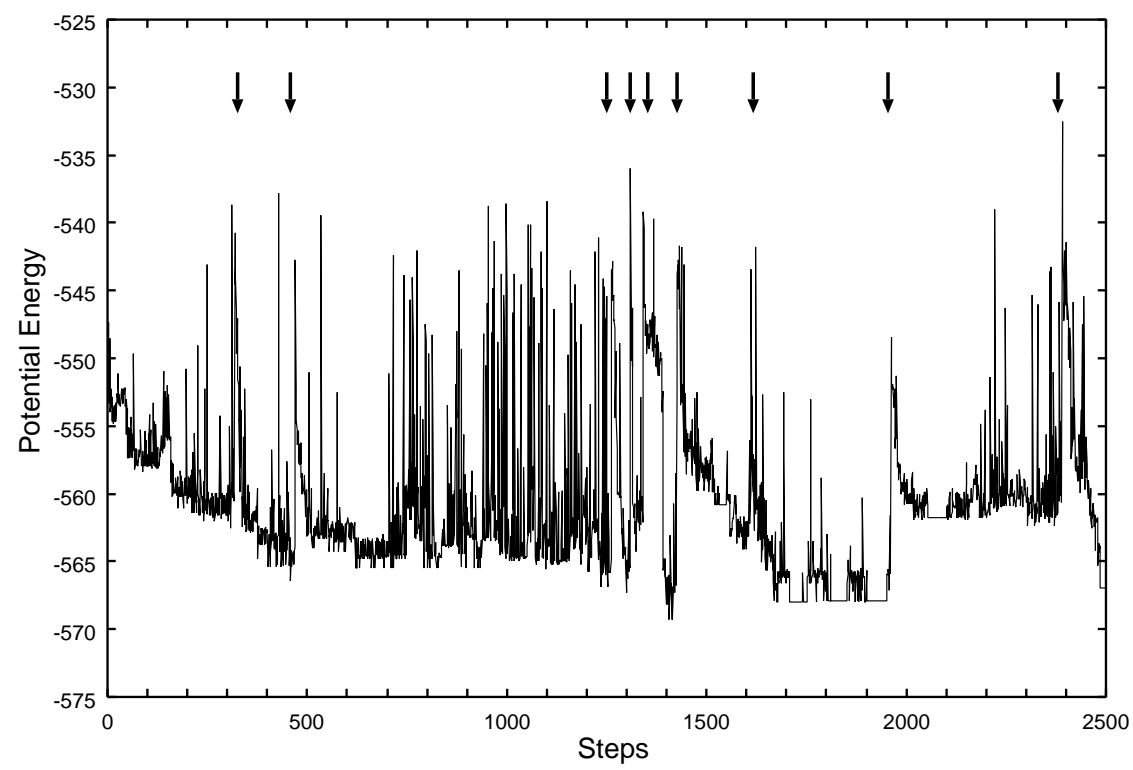

Fig. 4. An example of the trajectory of the successive run of BHOJ for $\mathrm{LJ}_{102}$. Arrows indicate the positions where the jumping occurs.

In comparison to the various sophisticated methods [21,22,23,24,25], our basinhopping with occasional jumping (BHOJ) is intuitively appealing and simple to implement. The performance of the algorithm seems better than most of the above algorithms.

Finally, in order to further test the performance of our BHOJ, we have used the BHOJ to search for the lowest-energy structure of larger clusters even larger than $\mathrm{LJ}_{148}[26,27]$.

Table 2

Success rates of original $\mathrm{BH}[5]$ and our BHOJ for selected larger Lennard-Jones clusters.

\begin{tabular}{crrr}
\hline Cluster & Energy & BH & BHOJ (JMP) \\
\hline $\mathrm{LJ}_{107}$ & -602.007110 & $12 / 100$ & $19 / 100(7)$ \\
$\mathrm{LJ}_{185}$ & -1125.493794 & $0 / 500$ & $1 / 500(5)$ \\
$\mathrm{LJ}_{186}$ & -1132.669966 & $1 / 200$ & $2 / 200(3)$ \\
$\mathrm{LJ}_{187}$ & -1139.455696 & $0 / 200$ & $2 / 200(3)$ \\
\hline
\end{tabular}

Table 2 shows the results for $\mathrm{LJ}_{107}$ and $\mathrm{LJ}_{185-187}$. For $\mathrm{LJ}_{107}$ we could confirm the new lowest-energy structure found by Wales and Doye [5] using our BHOJ. We could also successfully confirm the lowest energy -1125.493794 cited in [27] found by Leary for $\mathrm{LJ}_{185}$ which is lower than the previous record -1125.304876 found by Hartke [23,27]. The new lowest-energies -1132.669966 for $\mathrm{LJ}_{186}$ and -1139.455696 for $\mathrm{LJ}_{187}$ found by Hartke $[23,27]$ were also successfully located by our BHOJ though the success rates of these three cases were very low. 


\section{Conclusion}

In this paper we proposed a way to improve the performance of the basin hopping $(\mathrm{BH})$ algorithm by introducing the jumping in addition to the hopping. We call this new algorithm as the basin-hopping with occasional jumping (BHOJ). The jumping is a process of heating the system and raising the temperature to infinitely high which is attempted when the trajectory in the phase space is trapped at a local minimum. By jumping, the trajectory can climb up high barriers and can explore the next valley. Thus the exploratory ability

of the algorithm is enhanced.

Experiments on benchmark problem of the Lennard-Jones clusters, in particular, for notorious difficult sizes of 75 to 77 particles $\mathrm{LJ}_{75-77}$, of 98 particles $\mathrm{LJ}_{98}$, and of 102 to 104 particles $\mathrm{LJ}_{102-104}$ reveal that the proposed BHOJ is really superior to the original $\mathrm{BH}$.

This jumping is easy to implement, and consumes very little CPU resources. Any adaptive or scheduled jumping could be easily incorporated. The BHOJ with jumping will be helpful to search for the lowest-energy structures of larger clusters and more complex clusters with many body forces.

\section{Acknowledgments}

One of the author (M.I.) would like to thank Department of Physics, Tokyo Metropolitan University for providing him a visiting fellowship.

\section{References}

[1] I. Beichl and F. Sullivan, Computing in Science and Engineering 2 (2000) 65.

[2] S. Kirkpatrick, C.D. Gelatt, M.P. Vecchi, Science 220 (1983) 671.

[3] L.T. Wille, Chem. Phys. Lett. 133 (1987) 405.

[4] Z. Li, H.A. Scheraga, Proc. Natl. Acad. Sci. U.S.A. 84 (1987) 6611.

[5] D.J. Wales, J.P.K. Doye, J. Phys. Chem. A 101 (1997) 5111.

[6] R.H. Leary and J.P.K. Doye, Phys. Rev. E 60 (1999) R6320.

[7] R.H. Leary, J. Global. Opt. 18 (2000) 367.

[8] P. Boettcher and A. Percus, Artificial Intelligence 119 (2000) 275. 
[9] A.Möbius, A. Neklioudov, A. Díaz-Sánchez, K.H. Hoffmann, A. Fachat, M. Schreiber, Phys. Rev. Lett. 79 (1997) 4297.

[10] D. Frenkel, B. Smit, Understanding Molecular Simulation From Algorithm to Application, Academic Press, 1986.

[11] M.R. Hoare, P. Pal, Adv. Phys. 20 (1971) 161.

[12] J.A. Northby, J. Chem. Phys. 87 (1987) 6166.

[13] D.M. Deaven, N. Tit, J.R. Morris, K.M. Ho, Chem. Phys. Lett. 256 (1996) 195.

[14] J.A. Niesse, H.R. Mayne, J. Chem. Phys. 105 (1996) 8428.

[15] J.P.K. Doye, M.A. Miller, D.J. Wales, J. Chem. Phys. 111 (1999) 8417.

[16] M. Iwamatsu, Proc. 2002 IEEE World Congr. Comput. Intelligence. pp.1180, IEEE Press, 2002.

[17] L. Ingber, Mathl. Comput. Modelling 12 (1989) 967.

[18] D.J. Wales, program "gmin", http://www-wales.ch.cam.ac.uk/software.html.

[19] D.J. Wales, "Cambridge Cluster Database", http://www-doye.ch.cam.ac.uk/jon/structures/LJ/tables.150.html).

[20] W. Cai, H. Jiang, X. Shao, J. Chem. Inf. Comput. Sci. 42 (2002) 1099.

[21] M.D. Wolf and U. Landmann, J. Phys. Chem. A 102 (1998) 6129.

[22] J. Pillardy, A. Liwo, H.A. Scheraga, J. Phys. Chem. A 103 (1999) 9370.

[23] G. Hartke, J. Comput. Chem. 20 (1999) 1752.

[24] I. Rata, A.A. Shvartsburg, M. Horoi, T. Frauenheim, K.W.M. Siu, K.A. Jackson, Phys. Rev. Lett. 85 (2000) 546.

[25] J. Lee, I-H. Lee, J. Lee, Phys. Rev. Lett. 91 (2003) 080201.

[26] D. Romero, C. Barrón, S. Gómez, Comp. Phys. Commun. 123 (1999) 87.

[27] C. Barrón, http://www.vcl.uh.edu/ cbarron/LJ_ cluster /LJpottable.html (currently down). 